Accretion Phenomena and Related Outfiows, IAU Colloquium 163

ASP Conference Series, Vol. 121, 1997

D.T. Wickramasinghe, L. Ferrario, and G.V. Bicknell, eds.

\title{
X-Ray and Optical Observations of a New X-Ray Soft Intermediate Polar: RX J0512.2-3241
}

\author{
V. Burwitz, K. Reinsch, K. Beuermann ${ }^{2}$ \\ Universitäts-Sternwarte, Geismarlandstr. 11, Göttingen, Germany
}

\author{
H.-C. Thomas \\ MPI für Astrophysik, D-85740 Garching
}

The V 17.6 mag optical counterpart of the bright, soft, high-galactic latitude X-ray source RX J0512.2-3241 detected during the ROSAT All-Sky Survey, has been identified as a new, asynchronously rotating, magnetic cataclysmic variable (intermediate polar). The X-ray spectrum of RX J0512-32 is similar to that of polars, it shows a soft component with no intrinsic absorption and a blackbody temperature $\mathrm{k} T_{\mathrm{bb}} \sim 38 \mathrm{eV}$. From our optical follow-up $B$ and $V$ CCD photometry (cf. Fig. 1) we derive most probable spin and orbital periods of $(863.5 \pm 0.7) \mathrm{s}$ and $(3.45 \pm 0.03) \mathrm{h}$ respectively. A lower limit for the distance to the system is $d>740 \mathrm{pc}$. From this evidence we suggest that RX J0512-32 is a further member of the ROSAT discovered class of soft X-ray intermediate polars (for details see Burwitz et al., 1996, A\&A 310, L25). This still small class of systems (see Haberl and Motch 1995, A\&A 297, L37) has X-ray characteristics similar to those of low magnetic field polars and may be their long sought evolutionary progenitors.

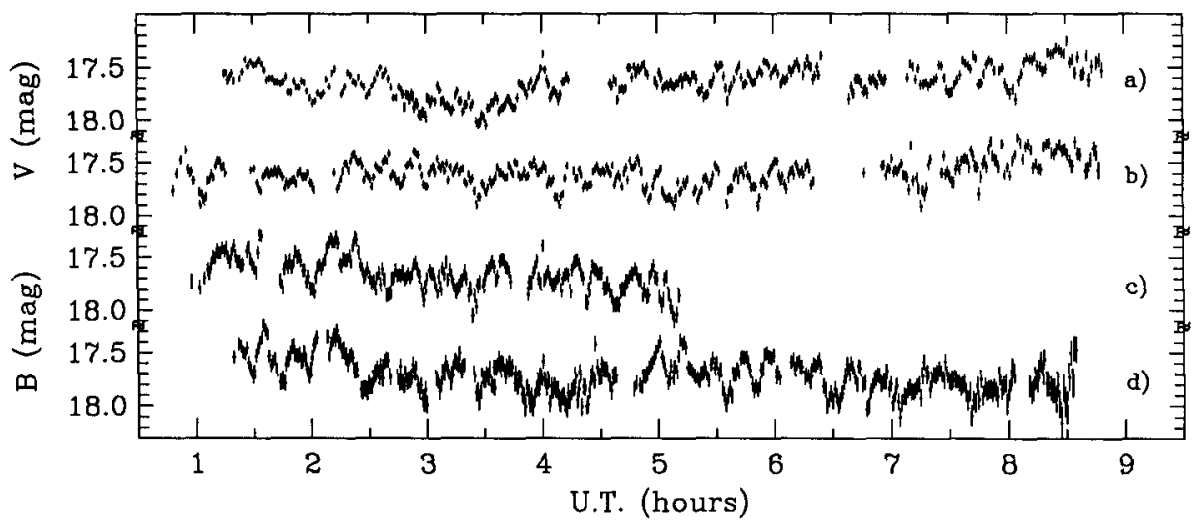

Figure 1. CCD photometry obtained with the $0.9 \mathrm{~m}$ ESO/Dutch telescope in $\mathrm{V}$ on a) Jan. 13, b) Jan. 14, and in B on c) Jan. 16, d) Jan. 17, 1996

\footnotetext{
${ }^{2}$ MPI für Extraterrestrische Physik, D-85740 Garching
} 